\title{
Dolor sacroilíaco: Revisión actualizada del enfrentamiento diagnóstico
}

\section{Sacroiliac Joint Pain: An Updated Review of the Clinical Approach for Diagnosis}

\author{
Enrique Blanco ${ }^{10}$ Sebastián Frías ${ }^{2}$ Facundo Álvarez ${ }^{3}$ José Fleiderman ${ }^{4}$
}

${ }^{1}$ Estudiante de Medicina, Universidad de Chile, Santiago, Chile

2 Residente Traumatología y Ortopedia, Clínica Universidad de los Andes, Hospital Militar de Santiago, Santiago, Chile

3 Fellow de Columna, Clínica Universidad de los Andes, Santiago, Chile

${ }^{4}$ Centro de columna, Traumatología y Ortopedia, Clínica Universidad de los Andes, Hospital del Trabajador. Universidad de los Andes, Santiago, Chile

\author{
Dirección para correspondencia Enrique Blanco Errázuriz, \\ Universidad de Chile, avenida Independencia 1.027, Independencia, \\ Región Metropolitana, Santiago, Chile \\ (e-mail: enriqueblancoe@gmail.com).
}

Rev Chil Ortop Traumatol 2021;62(2):e143-e152.

\section{Resumen \\ Palabras clave \\ - dolor sacroilíaco \\ - examen físico \\ - diagnóstico diferencial \\ - dolor lumbar bajo}

El dolor sacroilíaco es responsable de $15 \%$ a $30 \%$ de los cuadros de dolor lumbar bajo. El diagnóstico de esta patología es un desafío para el médico, debido a su compleja anatomía, el amplio diagnóstico diferencial, y las diversas etiologías que pueden provocar dolor en la articulación sacroilíaca. Una anamnesis ordenada y dirigida, asociada a un examen físico preciso, ayuda a orientar el diagnóstico. Las pruebas sacroiliacas específicas deben realizarse en aquellos pacientes con sospecha de dolor sacroilíaco, y deben interpretarse en conjunto y no de manera aislada. La resonancia magnética sirve para descartar otras causas de dolor lumbar bajo o diagnosticar casos de sacroileítis inflamatoria. La infiltración de la articulación es el gold standard para el diagnóstico, y debe realizarse en pacientes con alta sospecha de dolor sacroilíaco, por la anamnesis, examen físico, y tres o más pruebas sacroilíacas específicas positivas.

Sacroiliac pain accounts for $15 \%$ to $30 \%$ of low back pain conditions. Its diagnosis is a challenge for the physician due to its complex anatomy, the wide differential diagnoses list, and its several causes. Diagnosis requires a structured clinical history and an accurate physical examination. Specific sacroiliac physical examination tests should be performed in patients with suspected sacroiliac joint pain and interpreted together, not in isolation. Magnetic resonance imaging can rule out other causes of low back pain or diagnose inflammatory sacroiliitis. Joint infiltration is the gold standard for diagnosis, and it should be performed in patients with a high suspicion of sacroiliac joint pain based on anamnesis, physical examination, and three or more positive specific sacroiliac tests. recibido

September 2, 2020

aceptado

January 21, 2021
DOI https://doi.org/

$10.1055 / \mathrm{s}-0041-1728741$. ISSN 0716-4548.
(C) 2021. Sociedad Chilena de Ortopedia y Traumatologia. All rights reserved.

This is an open access article published by Thieme under the terms of the Creative Commons Attribution-NonDerivative-NonCommercial-License, permitting copying and reproduction so long as the original work is given appropriate credit. Contents may not be used for commercial purposes, or adapted, remixed, transformed or built upon. (https://creativecommons.org/ licenses/by-nc-nd/4.0/)

Thieme Revinter Publicações Ltda., Rua do Matoso 170, Rio de Janeiro, RJ, CEP 20270-135, Brazil 


\section{Introducción}

El dolor lumbar es la principal causa de dolor crónico no oncológico en Chile. ${ }^{1,2}$ Tiene consecuencias que impactan no solo en la persona que sufre el dolor, sino también en la población general, siendo un factor importante en el ausentismo laboral y licencias médicas, ${ }^{3}$ generando un importante costo económico para el país. Dentro de las etiologías del dolor lumbar, el dolor sacroilíaco (DSI) está descrito como el responsable en $15 \%$ a $30 \%$ de los casos. ${ }^{4} \mathrm{Sin}$ embargo, a pesar del impacto en la calidad de vida de los pacientes con esta patología, ${ }^{5}$ el DSI suele ser subdiagnosticado ante la presencia de dolor lumbar. ${ }^{6}$

En la actualidad, ya sea en atención primaria o en una consulta de traumatología general, es un verdadero desafío identificar la articulación sacroilíaca (ASI) como la responsable del dolor lumbar bajo. Esto se debe, entre otras razones, a su bajo índice de sospecha entre el personal médico, y a la baja especificidad y alta tasa de falsos positivos que presenta la anamnesis y el examen físico. $^{7} \mathrm{~A}$ estos motivos se les suman las siguientes razones:

1) Heterogénea manifestación clínica del dolor, variable en localización, irradiación e intensidad. ${ }^{8,9}$

2) Numerosa y diversa cantidad de pruebas especiales descritas para el examen físico, lo que refleja que ninguna por sí sola tiene suficiente aplicabilidad diagnóstica. ${ }^{10-13}$

3) La prevalente coexistencia con otras patologías degenerativas lumbares que podrían eventualmente ser hallazgos imagenológicos no sintomáticos, o potenciales fuentes de dolor. ${ }^{14}$

4) El amplio diagnóstico diferencial que podría justificar este dolor. ${ }^{15}$

5) Ausencia de recomendaciones o guías clínicas en nuestro país que propongan un enfrentamiento uniforme.

En el presente artículo, se presenta una revisión de la literatura con intención de proponer una aproximación diagnóstica al paciente con DSI, de manera tal de optimizar los recursos y mejorar el rendimiento diagnóstico. La revisión se hizo en base a la búsqueda en la plataforma PubMed "sacroiliac joint pain" asociado a las palabras clave "physical examination" y "diagnosis", filtrando por artículos de tipo Review, Systematic review, clinical trial y Meta-Analysis, obteniéndose 139 artículos, de los cuales se seleccionaron por su impacto y pertinencia según el criterio del equipo de autores. Revisaremos la anatomía, etiología, anamnesis, examen físico y exámenes complementarios para finalmente exponer una propuesta de algoritmo diagnóstico para aproximarnos al paciente que consulta por dolor lumbar bajo.

\section{Anatomía y biomecánica}

La ASI es la articulación axial más grande de nuestro esqueleto, y se encarga de distribuir la carga axial de la columna en ambas extremidades inferiores. Se trata de una articulación mixta, puesto que el tercio anterior de la superficie articular está compuesto por cartílago hialino y presenta cápsula articular, mientras que en sus dos tercios posteriores está compuesta por fibrocartílago y una densa red de ligamentos, que se comporta como una sindesmosis, ${ }^{16}$ como se aprecia en la - Figura 1.

La inervación de la ASI es un tema controvertido. ${ }^{17}$ Por posterior, está inervada por ramas laterales de la rama dorsal de L4-S3, mientras que por anterior se postula que estaría inervada por L2-S2. Sin embargo, dado la proximidad que existe con el plexo lumbosacro y músculos como el piriforme, glúteo máximo y el bíceps femoral, procesos inflamatorios en la ASI pueden llevar a irritación de estas estructuras e irradiación del dolor proveniente de la articulación. ${ }^{18,19}$

El movimiento de la ASI es extremadamente limitado, y varía según el sexo y etapa del ciclo vital del individuo. En niños y adolescentes, los ligamentos que la estabilizan son más flexibles, mientras que en la vejez esta articulación tiende a anquilosarse, especialmente en varones. ${ }^{19}$ Sus principales movimientos son la nutación y contranutación. En la primera, se produce una rotación del sacro en el plano sagital, llevando el extremo distal del sacro hacia posterior. Este movimiento es escaso en condiciones normales, siendo de alrededor de $1 \mathrm{~mm}$ a $4 \mathrm{~mm}$, pero aumenta considerablemente en la mujer embarazada para favorecer el parto vaginal. ${ }^{20}$ En el movimiento de contranutación, por

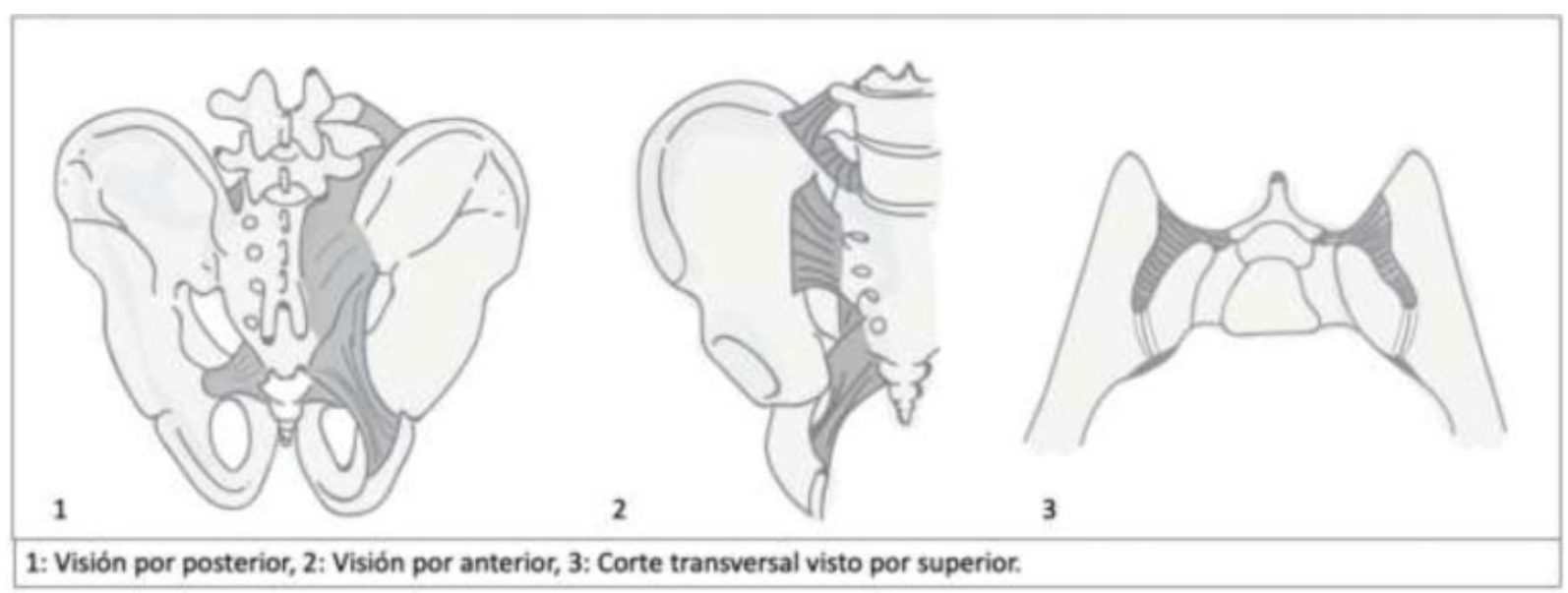

Fig. 1 Anatomía de las articulaciones sarcoilíacas (ASIs). 1: visión por posterior; 2: visión por anterior; 3: corte transversal visto por superior. 


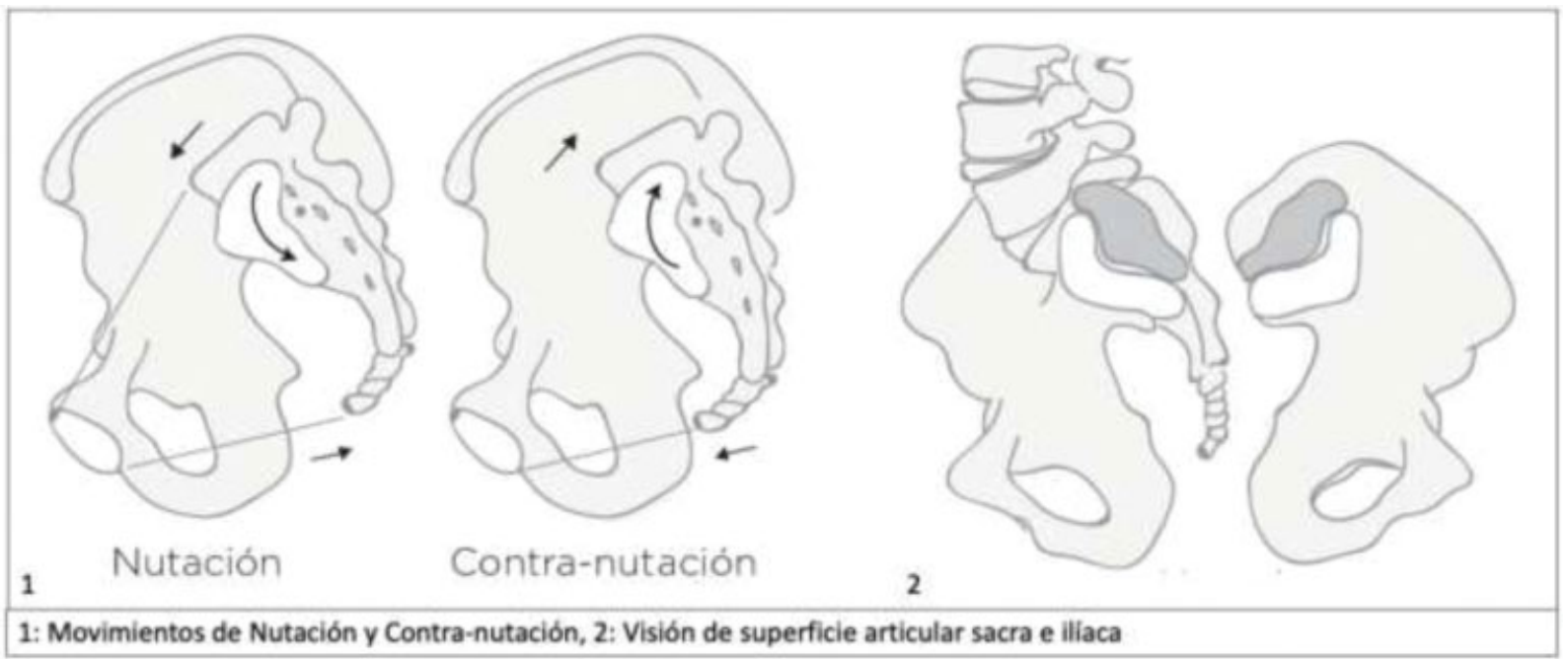

Fig. 2 Movimiento de las articulaciones sarcoilíacas (ASIs). 1: Movimiento de nutación y contranutación; 2: visión de superficie articular sacra e ilíaca.

otra parte, el sacro rota de manera tal que su porción distal se acerca a la sínfisis del pubis, mientras que su porción superior se dirige hacia posterior (-Figura 2).

Los componentes responsables de la estabilidad de la ASI son estructuras óseas y ligamentosas. La estabilidad vertical está sustentada en la estructura ósea que transmite las fuerzas de carga axial en compresión lateral hacia los huesos ilíacos y posteriormente las caderas, transformando el hueso sacro en la piedra angular de la arquitectura pelviana. El reforzado complejo ligamentoso sacroilíaco se encarga de la estabilidad en el plano anteroposterior (AP), ${ }^{21}$ como se aprecia en la - Figura 1.

\section{Etiología y diagnóstico diferencial del dolor sacroilíaco}

Al enfrentarse a un paciente con dolor lumbar bajo, el médico debe preguntarse si está efectivamente ante un DSI y no un dolor de otro origen. Durante la evaluación del paciente, se deben tener en mente todas las causas no sacroilíacas de un dolor lumbar bajo, tanto musculoesqueléticas, sobretodo la patología lumbar 0 de cadera, como viscerales o intrapélvicas, ya que todas ellas forman parte del diagnóstico diferencial. ${ }^{4,22-24}$ Las causas de dolor lumbar bajo se resumen en la - Tabla 1.

La ASI, por su parte, puede verse afectada por numerosas patologías, que pueden ser primarias o bien patologías sistémicas que de manera secundaria la afecten. En caso de las patologías primarias, las causas son eminentemente mecánicas o de origen infeccioso. ${ }^{24,25}$ Se ha descrito un cuadro que puede generar DSI que obedece a un fenómeno similar a lo que ocurre en un síndrome transicional en las cirugías de columna, en el que se genera una hipermovilidad de un segmento vertebral secundario a la fijación de segmentos adyacentes. Las ASIs también pueden desarrollar esta hipermovilidad luego de una artrodesis lumbosacra, desarrollando dolor. ${ }^{26-28}$ Las causas secundarias de DSI obedecen a enfermedades sistémicas, algunas de ellas de origen inflamatorio, como la espondilitis anquilosante, o bien metabólicas, como el hiperparatiroidismo. ${ }^{15,29}$ Las causas primarias y secundarias de DSI se resumen en la - Tabla 2.

\section{Historia clínica}

Para identificar correctamente el dolor, el médico debe tomar en consideración elementos anamnésicos importantes como la aparición, perfil temporal, carácter, factores gatillantes o atenuantes, irradiación, y localización. La mayoría de las

Tabla 1 Causas de dolor lumbar bajo $4,24-26$

\begin{tabular}{|l|}
\hline Patología lumbar: \\
\hline Discopatía lumbar \\
\hline Síndrome facetario \\
\hline Síndrome miofascial \\
\hline Artrosis de columna lumbar \\
\hline Patología de cadera: \\
\hline Artrosis de cadera \\
\hline Pinzamiento femoroacetabular \\
\hline Tendinitis glútea \\
\hline Bursitis trocantérica \\
\hline Dolor de la articulación sacroilíaca \\
\hline Causas intrapelvianas: \\
\hline Proceso inflamatorio pélvico \\
\hline Endometriosis \\
\hline Apendicitis retrocecal \\
\hline Diverticulitis \\
\hline Abscesos tuboováricos \\
\hline Cólico renal \\
\hline Neoplasia \\
\hline
\end{tabular}


Tabla 2 Causas de dolor sacroilíaco ${ }^{17,26-31}$

\begin{tabular}{|l|}
\hline Causas primarias \\
\hline Fractura previa \\
\hline Microtrauma \\
\hline Microinestabilidad por hiperlaxitud en el embarazo \\
\hline Sacroileítis infecciosa \\
\hline Osteomielitis crónica \\
\hline Hipermovilidad postartrodesis lumbosacra \\
\hline Osteitis condensante ilíaca \\
\hline Dolor sacroilíaco idiopático \\
\hline Causas secundarias \\
\hline Espondilitis anquilosante \\
\hline Artritis psoriática \\
\hline Artritis asociada a enfermedad inflamatoria intestinal \\
\hline Artritis reactiva \\
\hline Condrocalcinosis \\
\hline Hiperparatiroidismo \\
\hline Enfermedad de Reiter \\
\hline
\end{tabular}

veces, el dolor de la ASI se manifiesta como dolor lumbar mecánico, bajo la altura de L5, específicamente inferior a la espina ilíaca posterosuperior (EIPS). ${ }^{9,12}$ Estudios con infiltración sacroilíaca en pacientes asintomáticos muestran áreas de dolor $10 \mathrm{~cm}$ caudal y $3 \mathrm{~cm}$ lateral a la EIPS, ${ }^{8}$ pero también puede manifestarse como un dolor referido al territorio glúteo, uni o bilateral, dolor de muslo, inguinal, de pierna, e incluso dolor de pie. ${ }^{9,30}$ También se ha descrito dolor lumbar asociado a cambios de posición, como sentarse o ponerse de pie. ${ }^{31}$

Dentro de los antecedentes, se debe preguntar dirigidamente en la anamnesis por algunos elementos que podrían predisponer a desarrollar un cuadro de DSI: historia de trauma pelviano, antecedentes quirúrgicos, especialmente artrodesis lumbosacra, ${ }^{26}$ embarazo actual o reciente, deportes o trabajos manuales que impliquen cizallamiento o torsión pélvica, como son los deportes de carga, de contacto, o patinaje. ${ }^{32}$

No se debe nunca dejar de lado una completa anamnesis general según corresponda, en la que se puede averiguar sobre otros antecedentes mórbidos, quirúrgicos, e incluso historia familiar de enfermedades autoinmunes. Dado que la sacroileítis puede ocurrir como manifestación de una enfermedad subyacente, se debe indagar si se asocia o no a síntomas generales como fiebre, baja de peso, o bien síntomas específicos como poliartralgias, síntomas abdominales, ginecológicos o urológicos. ${ }^{24}$

En la - Tabla 3 se propone una serie de preguntas como primera aproximación al paciente, para poder descartar distintos diagnósticos diferenciales frente a dolor lumbar bajo. En caso de que alguna de las respuestas del paciente sea afirmativa, se debe pensar en causas de dolor no sacroilíacas,
Tabla 3 Abordaje para descartar dolor sacroilíaco en anamnesis

¿Localización atípica del dolor? (Cefálico a L5, anterolateral,
profundo o no objetivable)
¿Dolor con características no mecánicas? (Nocturno, duele
en reposo, no responde a antiinflamatorios no esteroideos)
¿Presencia de síntomas constitucionales? (Fiebre, baja de
peso, fatiga)
¿Presencia de síntomas gastrointestinales, urológicos, y/o
ginecológicos?

o bien que el dolor de esta articulación se deba a otra patología sistémica.

\section{Examen físico}

El examen físico en un paciente en el que se sospecha DSI debe ser completo y preciso. Dado que el tiempo en la consulta muchas veces es limitado, el examen físico se acota según la sospecha fundada en los elementos de la anamnesis. Sin embargo, en casos en que la historia clínica sea dudosa, se hace fundamental realizar un examen físico completo de cadera y de columna lumbar.

Se debe comenzar por un examen general, evaluando la marcha, posturas antiálgicas y deformidades evidentes, como asimetría significativa del largo de los miembros inferiores o escoliosis lumbar. ${ }^{24}$ Se debe continuar con la inspección y palpación de estructuras óseas y musculares al nivel de la cadera y de la región lumbar buscando puntos sensibles; incluso puede ser necesario buscar sensibilidad a la palpación abdominal, si se sospecha de un dolor referido de origen abdominopélvico.

Luego de la palpación es importante evaluar los rangos de movilidad tanto de la columna lumbar como de la cadera, evaluando movimientos activos y pasivos, y comparando con el lado contralateral. La evaluación motora y sensitiva sistemática de cada raíz nerviosa es de vital importancia para no pasar por alto algún déficit del que el paciente no se haya percatado.

Finalmente se deben realizar las pruebas de provocación de dolor radicular, pinzamiento femoroacetabular, y otras en las que se buscan signos de patología específica que forma parte del diagnóstico diferencial. ${ }^{23}$ En la -Tabla 4 se proponen elementos del examen físico que, de estar

Tabla 4 Abordaje para descartar dolor sacroilíaco en examen físico

Presencia de masa o dolor a la palpación abdominal.

Rango articular de cadera limitado o doloroso, palpación osteomuscular dolorosa.

Dolor a la palpación lumbar paravertebral o apófisis espinosas. Rango de movimientos lumbares dolorosos.

Signos de provocación radicular positivos, fuerza o sensibilidad alteradas en las extremidades inferiores. 
Tabla 5 Pruebas sacroilíacas de provocación de dolor $^{10-13,26,36-38}$

\begin{tabular}{|l|l|l|l|l|l|}
\hline Prueba* & Sensibilidad & Especificidad & Fiabilidad & $\begin{array}{l}\text { Valor predictivo } \\
\text { positivo }\end{array}$ & $\begin{array}{l}\text { Valor predictivo } \\
\text { negativo }\end{array}$ \\
\hline Distracción pélvica & $23-60 \%$ & $81-98 \%$ & $82 \%$ & $60-93 \%$ & $57-81 \%$ \\
\hline Compresión pélvica & $26-69 \%$ & $69-100 \%$ & $82-87 \%$ & $52-100 \%$ & $59-82 \%$ \\
\hline Compresión femoral (thigh thrust) & $36-88 \%$ & $69 \%$ & $84 \%$ & $58 \%$ & $92 \%$ \\
\hline Gaenslen & $31-71 \%$ & $71-94 \%$ & $82-88 \%$ & $47-81 \%$ & $60-77 \%$ \\
\hline Compresión sacra (sacral thrust) & $53 \%$ & $75 \%$ & $66-78 \%$ & $56 \%$ & $80 \%$ \\
\hline Prueba de Patrick (FABER) & $34-69 \%$ & $92 \%$ & $74-80 \%$ & $81 \%$ & $60 \%$ \\
\hline Drop test & - & - & $88-97 \%$ & - & - \\
\hline $\begin{array}{l}\text { Prueba de distracción de } \\
\text { la espina ilíaca posterosuperior }{ }^{37 * *}\end{array}$ & $100 \%$ & $89 \%$ & $94 \%$ & $90 \%$ & $100 \%$ \\
\hline
\end{tabular}

Abreviatura: FABER, flexion, abduction, external rotation (flexión, abducción, rotación externa).

Notas: *Se considera positiva con al menos un $80 \%$ de alivio de dolor postinfiltración anestésica. **En el estudio que propuso esta prueba, se consideró positivo con un $50 \%$ de alivio del dolor; por eso, presenta valores superiores al resto.

alterados, nos orientan a una causa del dolor distinta de la articulación sacroilíaca.

Cuando el examen físico realizado no orienta a patología coxal ni lumbar es cuando comienzan a tener utilidad las pruebas específicas de la ASI. Dada la alta tasa de falsos positivos que podrían presentar algunas de las pruebas sacroilíacas, se hace importante utilizarlas con discreción, sobretodo en presencia de patología coxal debido a que existen algunas pruebas descritas para patología sacroilíaca que también son utilizadas para el diagnóstico de patología coxal, incluso con mayor efectividad diagnóstica. ${ }^{33}$

Se han descrito diversas pruebas específicas el diagnóstico de DSI. ${ }^{7,10,12,13,34-36}$ Cuando tres de estas pruebas son positivas, el valor predictivo negativo (VPN) para DSI alcanza un $87 \%{ }^{37}$ Las pruebas más utilizadas en la práctica clínica se describen a continuación; en la - Tabla 5 se presenta un resumen las pruebas sacroilíacas (que se muestran en la -Figura 3), y se indica su sensibilidad, especificidad, fiabilidad, VPN y valor predictivo positivo (VPP).

Prueba de Gaenslen: paciente en decúbito supino. Se le pide que flecte cadera y rodilla del lado afectado, lleve la rodilla hacia su pecho y la sostenga con sus brazos. La pierna contralateral debe dejarla colgando por el borde de la camilla. El examinador presiona la rodilla flectada hacia el pecho, y ejerce una contrapresión en la otra rodilla. La prueba se considera positiva si reproduce el dolor exacto.

Compresión sacra: paciente en decúbito prono. El examinador ejerce presión sobre el sacro. La prueba se considera positiva cuando se reproduce el dolor exacto.

Compresión femoral: paciente en posición supina, con la cadera del lado afectado flectada en $90^{\circ}$ y rodilla ipsilateral también flectada. El examinador coloca una mano en el sacro del paciente, y con la otra sostiene la rodilla flectada. Aduce levemente la cadera y ejerce fuerza a través del eje principal del fémur, hacia el sacro. La prueba se considera positiva cuando se reproduce el dolor exacto del paciente.
Compresión pélvica: paciente en decúbito lateral, con el lado afectado hacia arriba, con flexión de caderas de $45^{\circ}$ y de rodillas de $90^{\circ}$. El examinador ejerce presión sobre la cresta ilíaca hacia abajo. La prueba se considera positiva si reproduce el dolor exacto.

Distracción pélvica: paciente en decúbito supino. El examinador aplica fuerza sobre ambas espinas ilíacas anterosuperiores, en dirección posterolateral. La prueba se considera positiva si reproduce el dolor exacto.

Prueba de Patrick (flexión, abducción, rotación externa; flexion, abduction, external rotation, FABER): paciente en decúbito supino. Se coloca el tobillo del lado que se quiere evaluar del paciente sobre su muslo contralateral, mediante flexión de rodilla, y abducción y rotación externa de la cadera ipsilateral. Luego, se ejerce presión sobre la rodilla flectada, conteniendo ambas espinas ilíacas anterosuperiores. El examen se considera positivo si el paciente refiere el mismo dolor en el lado ipsilateral.

Test del dedo de Fortin: paciente en posición de pie. Se le pide que indique con un dedo el punto de mayor dolor, dos veces. Si en ambas oportunidades indica un punto $2 \mathrm{~cm}$ inferomedial a la espina iliaca posterosuperior, se considera positivo.

Prueba de caída (drop test): paciente en monopedestación sobre el pie del lado afectado. Se le pide que se realice apoyo metatarsal (en puntillas) y se deje caer sobre el talón. La prueba se considera positiva si se reproduce el dolor.

Prueba de Gillet: paciente de pie, el examinador se posiciona detrás. Coloca un pulgar sobre la espina ilíaca posterosuperior del lado afectado, y el otro sobre el proceso espinoso de S2, a la misma altura. Solicita al paciente flectar la cadera ipsilateral. Lo normal es que la espina ilíaca posterosuperior descienda $1 \mathrm{~cm}$ o $2 \mathrm{~cm}$. Se considera positiva si la espina no desciende, reflejando hipomovilidad de la ASI.

Test de distracción de espina ilíaca posterosuperior: ${ }^{35}$ paciente de pie o en decúbito prono. Se colocan ambos pulgares en la espina ilíaca posterosuperior, y se ejerce 

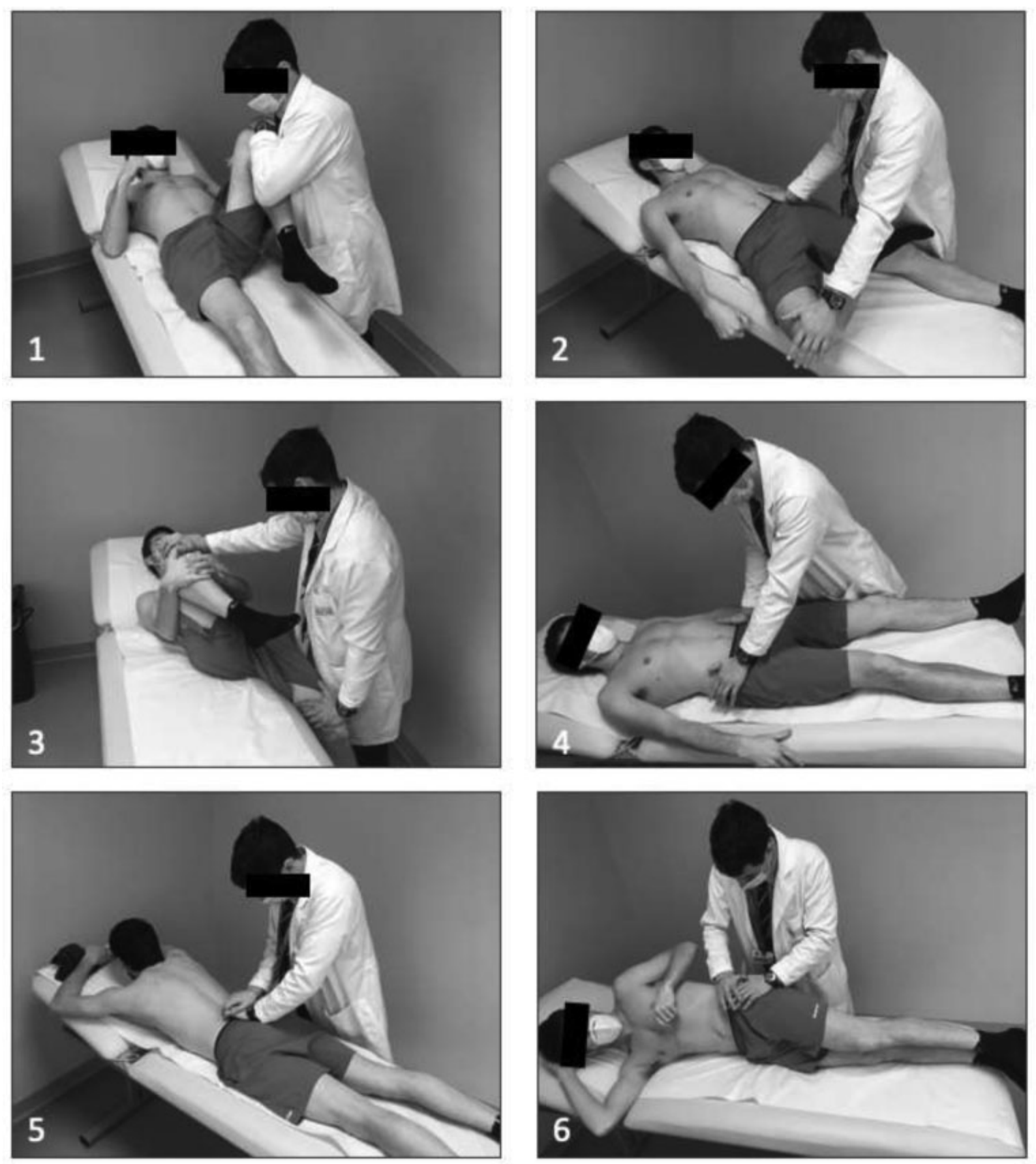

\section{1: Compresión femoral, 2: FABER, 3: Gaenslen, 4: Distracción pélvica, 5: Compresión sacra, 6: Compresión pélvica}

Fig. 3 Pruebas de provocación de dolor sacroilíaca. 1: Compresión femoral; 2: FABER; 3: Gaenslen; 4: distracción pélvica; 5: compresión sacra; 6: compresión pélvica. Abreviatura: FABER, flexion, abduction, external rotation (flexión, abducción, rotación externa).

presión de medial a lateral en cada una. La prueba se considera positiva si se reproduce el dolor.

\section{Imágenes}

La utilización de imágenes para el diagnóstico de DSI es un tema controversial. Por un lado, las patologías que afectan a la ASI pueden no tener manifestación radiológica, o bien una misma enfermedad puede tener manifestaciones diversas. ${ }^{29}$ Por otra parte, diversas estructuras anatómicas pueden verse alteradas en las imágenes, sin ser necesariamente causantes del dolor del paciente, lo que puede llevar a errores diagnósticos. En un estudio realizado por Boden et al., ${ }^{14}$ se estudiaron mediante resonancia nuclear magnética a 67 
individuos asintomáticos, se encontraron anormalidades sustanciales en la columna lumbar en un tercio de ellos, cifra que se eleva a un $57 \%$ en el grupo mayor de 60 años. De manera similar, en un estudio llevado a cabo por Eno et al. ${ }^{38}$ en 2015 , se estudiaron retrospectivamente tomografías computarizadas de 373 pacientes sin dolor lumbar bajo ni de la cintura pélvica, para analizar las ASI. Encontraron que un $65.1 \%$ de los pacientes tenía signos de degeneración de la ASI, lo que aumentaba con la edad, llegando a un $100 \%$ en mayores de 90 años.

En caso de no tener certeza respecto de que el dolor del paciente provenga de las ASIs, algunos autores plantean razonable solicitar una radiografía de pelvis AP que, en conjunto con la historia clínica y el examen físico, puede orientar al diagnóstico diferencial. ${ }^{24}$ Sin embargo, este tipo de estudio en particular resulta poco sensible, y no existe evidencia de calidad que lo respalde. Si el índice de sospecha comprende la presencia de síntomas de alarma como edades extremas, cronificación del dolor, dolor invalidante, trauma reciente, déficit neurológico, historia de cáncer, uso de corticoides, u otros, está indicado solicitar una resonancia magnética según la sospecha diagnóstica, que presenta una mayor sensibilidad y especificidad. ${ }^{2}$ La resonancia magnética de las ASIs permite apreciar con mayor precisión la articulación, e identificar cambios inflamatorios precoces y alteraciones estructurales, sobretodo en pacientes con pelviespondilopatías, ${ }^{39}$ y la resonancia magnética lumbar a su vez permite buscar un origen distinto del dolor. ${ }^{24}$ Otro examen imagenológico utilizado en la práctica clínica es el cintigrama óseo, ${ }^{40}$ que otorga la ventaja de ver el cuerpo completo, lo cual es útil frente a cuadros inespecíficos con hipótesis diagnósticas amplias. Se ha descrito que es especialmente útil en caso de no tener acceso a una infiltración diagnóstica o cuando el diagnóstico es poco claro, y que podría indicar un origen mecánico del dolor. ${ }^{41}$

\section{Infiltración diagnóstica}

Debido a la falta de precisión diagnóstica tanto de las imágenes como de las pruebas específicas de provocación sacroilíacas, puede ser necesario en algunos casos utilizar la infiltración diagnóstica como herramienta, la cual es considerada el gold standard para el diagnóstico de DSI. $^{42}$ La infiltración diagnóstica tiene varias ventajas. En primer lugar, permite confirmar o descartar la fuente del dolor, lo que a su vez permite determinar si el paciente se beneficiaría de una eventual artrodesis sacroilíaca. Además, es un procedimiento simple y con escasas complicaciones. Por último, no solo va a tener utilidad diagnóstica, sino que muchas veces puede también ser terapéutica; a pesar de que no exista evidencia de calidad que justifique su uso, la infiltración sacroilíaca terapéutica es una práctica que se realiza cada vez con mayor frecuencia en los Estados Unidos. ${ }^{43}$

Para que este procedimiento sea efectivo y válido o interpretable, se requiere de 2 condiciones fundamentales: 1) la infiltración debe ser realizada en pabellón, guiada por radiografía intraoperatoria, corroborando bajo visión directa que el anestésico con contraste se deposite en espacio intraarticular, pues se ha descrito que la punción ciega tiene solo un $22 \%$ de éxito; ${ }^{44}$ y 2 ) se deben infiltrar un volumen máximo de $1 \mathrm{~mL}$ a $1,5 \mathrm{~mL}$ de anestésico local y corticoides, pues si se inyectan volúmenes mayores, el líquido puede difundir hacia otros territorios y generar falsos positivos. ${ }^{8}$

El procedimiento debe realizarse con la técnica adecuada. ${ }^{45}$ Primero se le realizan las pruebas específicas y se le pide al paciente que le ponga nota de 1 a 100 a la intensidad de cada una de las pruebas. Luego, se posiciona al paciente en decúbito prono, se realiza una radiografía AP guiando la punción hacia el receso inferior de la ASI, se marcan $1 \mathrm{~cm}$ a $2 \mathrm{~cm}$ superior al aspecto más inferior de la ASI, y se realiza la punción orientando la dirección de la aguja desde medial hacia lateral, a medida que se va corroborando con variaciones oblicuas de la radiografía AP con angulaciones hacia cefálico, caudal o laterales. Una vez confirmado que la punta de la aguja se encuentra intraarticular en dos proyecciones ortogonales, se procede a inyectar la solución de contraste con anestésico, corroborando bajo rayos que se infiltró el espacio correspondiente sin la existencia de fuga. Finalmente, se procede a repetir las pruebas específicas y registrar la intensidad del dolor para cada una de ellas.

Varios autores ${ }^{31,37}$ han propuesto que, para minimizar el número de infiltraciones diagnósticas, estas sean realizadas en aquellos pacientes que tienen tres o más pruebas de provocación positivas, ya que si tiene menos de tres, la probabilidad de que el dolor sea sacroilíaco es baja. La realización de pruebas de provocación de dolor posterior a la inyección es un indicador que ayuda a distinguir si el dolor proviene o no de la ASI. ${ }^{46}$ Normalmente se considera positiva una infiltración si el alivio del dolor tras el procedimiento supera el $50 \%$ o incluso el $75 \%$. $^{37,47}$

\section{Discusión}

El diagnóstico clínico del DSI no es sencillo, debido a la compleja anatomía de la articulación y la diversidad de causas que lo pueden explicar. Una adecuada anamnesis y examen físico permiten orientar el estudio diagnóstico, sospechar diferentes causas anatómicas del dolor o bien causas sistémicas de DSI, y definir si corresponde o no hacer las pruebas sacroilíacas. Las pruebas sacroilíacas, por su parte, han sido objeto de investigación debido a su controversial rol diagnóstico.

Fortin et al. ${ }^{8,9}$ demostraron en 1994 el patrón de localización del DSI. En un estudio posterior, Fortin y Falco $^{12}$ describieron el "Test del dedo de Fortin" como una prueba exitosa para el diagnóstico de DSI. Sin embargo, algunos autores han postulado que las pruebas de provocación de dolor de la ASI son más fiables que la palpación. ${ }^{34,48}$ Robinson et al., ${ }^{34}$ en un estudio realizado en 2007 en 61 pacientes con sospecha de DSI, concluyeron que las pruebas de palpación tienen índices de concordancia entre examinadores significativamente menores que las pruebas de provocación. Por otra parte, la utilidad de las pruebas sacroilíacas de provocación de dolor realizadas de 


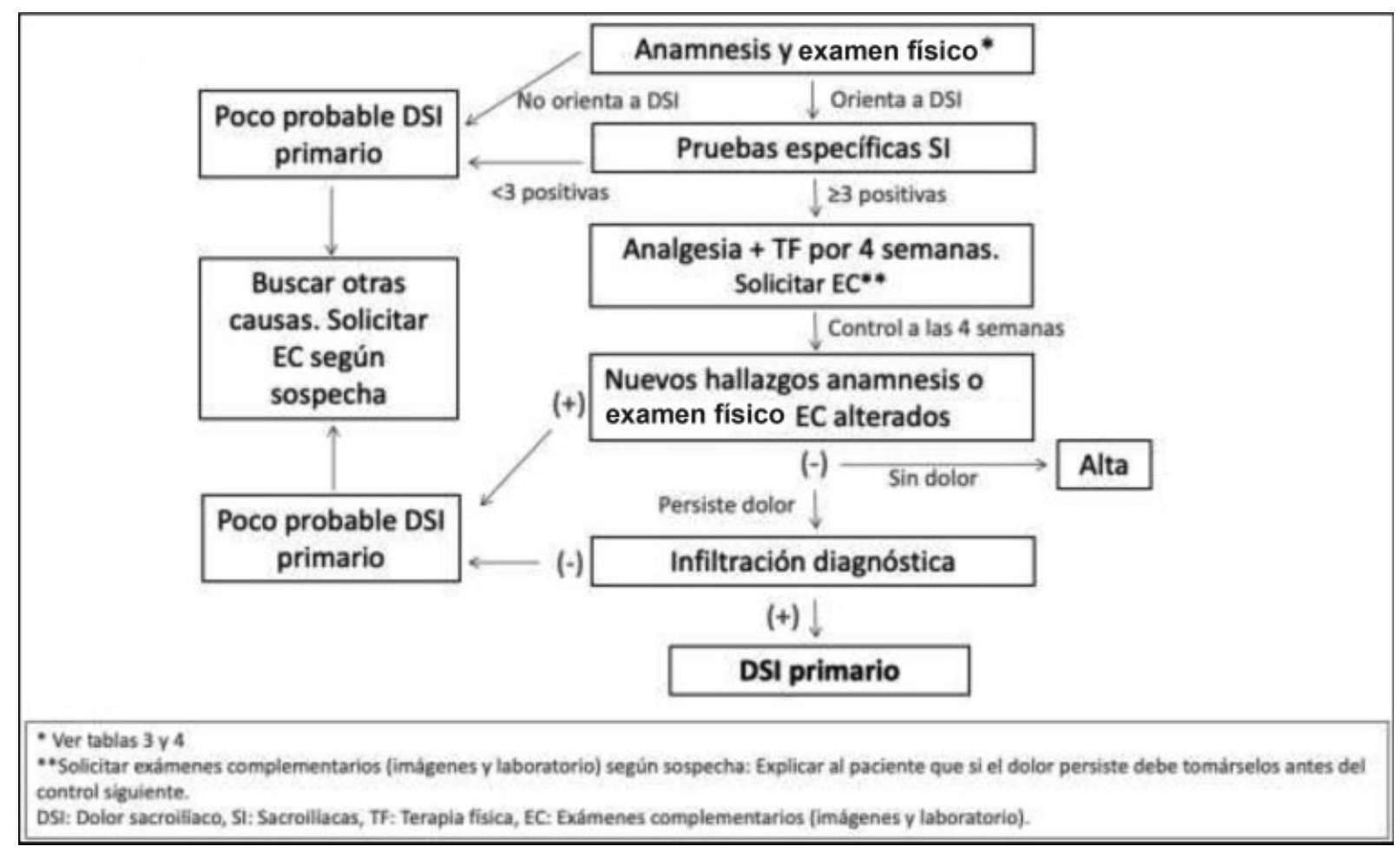

Fig. 4 Algoritmo de enfrentamiento diagnóstico de dolor sacroilíaco en paciente con dolor lumbar bajo. Adaptado de Thawrani DP, Agabegi SS, Asghar F. Diagnosing Sacroiliac Joint Pain: J Am Acad Orthop Surg. 2019; 27: 85-93, y modificado por los autores según experiencia clínica.

manera aislada también ha sido cuestionada, debido a su baja especificidad, sensibilidad, y alta tasa de falsos positivos. ${ }^{49,50}$ Hasta la actualidad, no se ha encontrado ninguna maniobra que tenga alguna aplicabilidad diagnóstica por sí sola, y se han reportado varias pruebas específicas con diversos indicadores de sensibilidad, especificidad $\mathrm{y}$ valores predictivos. $^{10-13,34-36}$

Diversos autores han postulado que la realización de más de una prueba y la interpretación de una combinación de estas serían una manera fiable de llegar al diagnóstico de DSI. ${ }^{13,34,37,51}$ En 2006, un estudio realizado por van der Wurff et al. ${ }^{37}$ en 60 pacientes con historia de dolor lumbar bajo demostraron, mediante la inyección de bloqueos articulares en pacientes sintomáticos y la realización de pruebas de provocación de dolor, que la presencia de menos de 3 pruebas positivas tiene un alto valor predictivo negativo (87\%), mientras que 3 o más pruebas específicas de provocación positivas tienen un alto valor predictivo positivo para DSI (65\% a 93\%). Kokmeyer et al., ${ }^{13}$ en un estudio realizado en 78 pacientes, llegan a conclusiones similares. Señalan que si de un conjunto de cinco pruebas de provocación de dolor, tres de ellas son negativas, el valor predictivo negativo es superior al de la realización de una prueba aislada, y que tiene mayor concordancia entre examinadores. Debido a estos últimos dos trabajos, se logró finalmente validar la utilidad clínica de las pruebas específicas sacroilíacas, y se concluyó que estas cumplen su rol cuando se realizan en conjunto.

Las pruebas sacroilíacas deben ser interpretadas correctamente en cada paciente en particular, según la sospecha diagnóstica que nos sugieran la anamnesis y el examen físico. Es por esta razón que la propuesta de realizar una anamnesis y examen físico sistemático previo a las pruebas específicas es de vital importancia, y permite seleccionar adecuadamente a aquellos pacientes con DSI y quienes se beneficiarían de procedimientos más invasivos, como la infiltración. En la - Figura 4 sugerimos un algoritmo que resume el enfrentamiento diagnóstico del dolor lumbar bajo en un contexto de sospecha de DSI, y en la - Tabla 6 presentamos una lista de exámenes complementarios que pueden solicitarse según la sospecha clínica.

Tabla 6 Exámenes complementarios

\begin{tabular}{|l|}
\hline Imágenes \\
\hline Resonancia magnética sacroilíaca \\
\hline Resonancia magnética lumbosacra \\
\hline Resonancia magnética de cadera \\
\hline Radiografías de pelvis y cadera \\
\hline Radiografía de columna lumbar \\
\hline Cintigrafía ósea \\
\hline Laboratorio \\
\hline $\begin{array}{l}\text { Laboratorio general: Hemograma, velocidad de hemosedi- } \\
\text { mentación (VHS), proteína c-reactiva (PCR), perfil bioquí- } \\
\text { mico, función renal }\end{array}$ \\
\hline $\begin{array}{l}\text { Laboratorio reumatológico: antígeno leucocitario humano } \\
\text { (human leukocyte antigen, HLA) B27, anticuerpo antinuclear } \\
\text { (ANA), anticuerpo nuclear extractable (extractable nuclear } \\
\text { antigen, ENA), factor reumatoide (FR), anticuerpo antipép- } \\
\text { tido cíclico citrulinado (anti-CCP) }\end{array}$ \\
\hline
\end{tabular}




\section{Conclusión}

El DSI es una causa importante de dolor lumbar, que puede ser proveniente de diversas patologías y suele ser subdiagnosticado. Su diagnóstico específico es un desafío para el médico, por lo que la aproximación ordenada $\mathrm{y}$ secuencial a este tipo de pacientes es fundamental. Para lograr un diagnóstico preciso, se requiere una anamnesis y un examen físico detallados que ayuden a descartar los principales diagnósticos diferenciales. La realización de varias pruebas sacroilíacas específicas es una herramienta útil en el contexto de un paciente con sospecha clara de DSI siempre que se encuentren descartadas otras fuentes probables de dolor, ya que tienen una alta tasa de falsos positivos. Es por el mismo motivo que la utilización combinada de estas pruebas optimiza su rendimiento diagnóstico, alcanzando VPNs de hasta un 87\%. Las imágenes no suelen ser necesarias para el diagnóstico, pero deben ser consideradas para estudiar otras causas del dolor especialmente en situaciones en que se encuentren signos de alarma. La infiltración diagnóstica de la articulación es el gold standard, y debe reservarse para pacientes en los que persista la sospecha de DSI, y puede ser tanto diagnóstica como parte del tratamiento.

\section{Conflicto de intereses}

Los autores declaran no haber conflicto de intereses.

\section{Referencías}

1 Miranda J, Quezada P, Caballero P, et al. Revisión Sistemática: Epidemiología de Dolor Crónico No Oncológico en Chile. El Dolor. 2013;59:10-17

2 Bilbeny N. Dolor Crónico en Chile. Rev Med Clin Las Condes 2019; 30:397-406

3 Diaz-Ledezma C, Urrutia J, Romeo J, Chelen A, González-Wilhelm L, Lavarello C. Factors associated with variability in length of sick leave because of acute low back pain in Chile. Spine J 2009;9(12): 1010-1015

4 Sembrano JN, Polly DW Jr. How often is low back pain not coming from the back? Spine 2009;34(01):E27-E32

5 Cher D, Polly D, Berven S. Sacroiliac joint pain: burden of disease. Med Devices (Auckl) 2014;7:73-81

6 Weksler N, Velan GJ, Semionov M, et al. The role of sacroiliac joint dysfunction in the genesis of low back pain: the obvious is not always right. Arch Orthop Trauma Surg 2007;127(10):885-888

7 Dreyfuss P, Michaelsen M, Pauza K, McLarty J, Bogduk N. The value of medical history and physical examination in diagnosing sacroiliac joint pain. Spine 1996;21(22):2594-2602

8 Fortin JD, Dwyer AP, West S, Pier J. Sacroiliac joint: pain referral maps upon applying a new injection/arthrography technique. Part I: Asymptomatic volunteers. Spine 1994;19(13):1475-1482

9 Fortin JD, Aprill CN, Ponthieux B, Pier J. Sacroiliac joint: pain referral maps upon applying a new injection/arthrography technique. Part II: Clinical evaluation. Spine 1994;19(13): 1483-1489

10 Laslett M, Aprill CN, McDonald B, Young SB. Diagnosis of sacroiliac joint pain: validity of individual provocation tests and composites of tests. Man Ther 2005;10(03):207-218

11 Dreyfuss P, Cole AJ, Pauza K. Sacroiliac Joint Injection Techniques. Phys Med Rehabil Clin N Am 1995;6:785-813

12 Fortin JD, Falco FJ. The Fortin finger test: an indicator of sacroiliac pain. Am J Orthop 1997;26(07):477-480
13 Kokmeyer DJ, Van der Wurff P, Aufdemkampe G, Fickenscher TCM. The reliability of multitest regimens with sacroiliac pain provocation tests. J Manipulative Physiol Ther 2002;25(01): $42-48$

14 Boden SD, Davis DO, Dina TS, Patronas NJ, Wiesel SW. Abnormal magnetic-resonance scans of the lumbar spine in asymptomatic subjects. A prospective investigation. J Bone Joint Surg Am 1990; 72(03):403-408

15 Campos-Correia D, Sudoł-Szopińska I, Diana Afonso P. Are We Overcalling Sacroillitis on MRI? Differential diagnosis that every rheumatologist should know - Part II. Acta Reumatol Port 2019; 44(01):42-56

16 Puhakka KB, Melsen F, Jurik AG, Boel LW, Vesterby A, Egund N. MR imaging of the normal sacroiliac joint with correlation to histology. Skeletal Radiol 2004;33(01):15-28

17 Vleeming A, Schuenke MD, Masi AT, Carreiro JE, Danneels L, Willard FH. The sacroiliac joint: an overview of its anatomy, function and potential clinical implications. J Anat 2012;221 (06):537-567

18 Fortin JD, Tolchin RB. Sacroiliac arthrograms and postarthrography computerized tomography. Pain Physician 2003;6 (03):287-290

19 Ares JDA, Amatriaín GR, González AP, Nieto C. Bloqueo y radiofrecuencia de la articulación sacroilíaca. Rev Soc Esp Dolor. 2012;19:335-345

20 Le Huec JC, Tsoupras A, Leglise A, Heraudet P, Celarier G, Sturresson B. The sacro-iliac joint: A potentially painful enigma. Update on the diagnosis and treatment of pain from microtrauma. Orthop Traumatol Surg Res 2019;105(1S):S31-S42

21 Kiapour A, Joukar A, Elgafy H, Erbulut DU, Agarwal AK, Goel VK. Biomechanics of the Sacroiliac Joint: Anatomy, Function, Biomechanics, Sexual Dimorphism, and Causes of Pain. Int J Spine Surg 2020;14(Suppl 1):3-13

22 Simons DG, Travell JG. Myofascial origins of low back pain. 1. Principles of diagnosis and treatment. Postgrad Med 1983;73 (02):66-77, 68-70, 73 passim

23 Molina M, Campos M, Delgado M. Lumbago: Revisión actualizada del diagnóstico, tratamiento y pronóstico. Rev Chil Ortop Traumatol. 2014;55:113-125

24 Thawrani DP, Agabegi SS, Asghar F. Diagnosing Sacroiliac Joint Pain. J Am Acad Orthop Surg 2019;27(03):85-93

25 Mitra R. Osteitis Condensans Ilii. Rheumatol Int 2010;30(03): 293-296

26 Ha K-Y, Lee J-S, Kim K-W. Degeneration of sacroiliac joint after instrumented lumbar or lumbosacral fusion: a prospective cohort study over five-year follow-up. Spine 2008;33(11):1192-1198

27 Faundez AA, Richards J, Maxy P, Price R, Léglise A, Le Huec J-C. The mechanism in junctional failure of thoraco-lumbar fusions. Part II: Analysis of a series of PJK after thoraco-lumbar fusion to determine parameters allowing to predict the risk of junctional breakdown. Eur Spine J 2018;27(Suppl 1):139-148

28 Charles YP, Yu B, Steib J-P. Sacroiliac joint luxation after pedicle subtraction osteotomy: report of two cases and analysis of failure mechanism. Eur Spine J 2016;25(Suppl 1):63-74

29 Pozo P. Enfermedades reumatológicas más frecuentes en atención primaria: Espondiloartritis. En:Pacheco D, editor. Reumatología para médicos de Atención Primaria. Segunda edición. Chile: Tangram Ediciones; 2015:167-180

30 Slipman CW, Jackson HB, Lipetz JS, Chan KT, Lenrow D, Vresilovic EJ. Sacroiliac Joint Pain Referral Zones. 2000;81:334-338

31 Ou-Yang DC, York PJ, Kleck CJ, Patel VV. Diagnosis and Management of Sacroiliac Joint Dysfunction. J Bone Joint Surg Am 2017;99(23):2027-2036

32 Correa-Illanes G. Dolor sacroilíaco: Epidemiología, fisiopatología, diagnóstico y tratamiento. Rehabil Integral. 2019;14:91-101

33 Martin RL, Sekiya JK. The interrater reliability of 4 clinical tests used to assess individuals with musculoskeletal hip pain. J Orthop Sports Phys Ther 2008;38(02):71-77 
34 Robinson HS, Brox JI, Robinson R, Bjelland E, Solem S, Telje T. The reliability of selected motion- and pain provocation tests for the sacroiliac joint. Man Ther 2007;12(01):72-79

35 Werner CML, Hoch A, Gautier L, König MA, Simmen H-P, Osterhoff G. Distraction test of the posterior superior iliac spine (PSIS) in the diagnosis of sacroiliac joint arthropathy. BMC Surg 2013;13:52-57

36 Stuber KJ. Specificity, sensitivity, and predictive values of clinical tests of the sacroiliac joint: a systematic review of the literature. J Can Chiropr Assoc 2007;51(01):30-41

37 van der Wurff P, Buijs EJ, Groen GJ. A multitest regimen of pain provocation tests as an aid to reduce unnecessary minimally invasive sacroiliac joint procedures. Arch Phys Med Rehabil 2006;87(01):10-14

38 Eno J-JT, Boone CR, Bellino MJ, Bishop JA. The prevalence of sacroiliac joint degeneration in asymptomatic adults. J Bone Joint Surg Am 2015;97(11):932-936

39 Poddubnyy D, Gaydukova I, Hermann K-G, et al. Magnetic resonance imaging compared to conventional radiographs for detection of chronic structural changes in sacroiliac joints in axial spondyloarthritis. J Rheumatol 2013;40(09):1557-1565

40 Zilber K, Gorenberg M, Rimar D, et al. Radionuclide Methods in the Diagnosis of Sacroiliitis in Patients with Spondyloarthritis: An Update. Rambam Maimonides Med J 2016;7(04):e0037

41 Maigne JY, Boulahdour H, Chatellier G. Value of quantitative radionuclide bone scanning in the diagnosis of sacroiliac joint syndrome in 32 patients with low back pain. Eur Spine J 1998;7 (04):328-331

42 Rupert MP, Lee M, Manchikanti L, Datta S, Cohen SP. Evaluation of sacroiliac joint interventions: a systematic appraisal of the literature. Pain Physician 2009;12(02):399-418
43 Manchikanti L, Hansen H, Pampati V, Falco FJE. Utilization and growth patterns of sacroiliac joint injections from 2000 to 2011 in the medicare population. Pain Physician 2013;16(04): E379-E390

44 Rosenberg JM, Quint TJ, de Rosayro AM. Computerized tomographic localization of clinically-guided sacroiliac joint injections. Clin J Pain 2000;16(01):18-21

45 Schwartz A, Zlomislic V, Reckling WC, Cher D. Sacroiliac Joint Pain: Pathophysiology and Diagnosis. En:Rothman RH, Simeone FA, Herkowitz HN, editores. Rothman-Simeone and Herkowitz's, The Spine. Seventh edition. Philadelphia, PA: Elsevier; 2018: 383-396

46 Polly DW Jr. The Sacroiliac Joint. Neurosurg Clin N Am 2017;28 (03):301-312

47 Simopoulos TT, Manchikanti L, Singh V, et al. A systematic evaluation of prevalence and diagnostic accuracy of sacroiliac joint interventions. Pain Physician 2012;15(03):E305-E344

48 Potter NA, Rothstein JM. Intertester reliability for selected clinical tests of the sacroiliac joint. Phys Ther 1985;65(11): $1671-1675$

49 van der Wurff P, Hagmeijer RH, Meyne W. Clinical tests of the sacroiliac joint. A systematic methodological review. Part 1: Reliability. Man Ther 2000;5(01):30-36

50 Berthelot J-M, Labat J-J, Le Goff B, Gouin F, Maugars Y. Provocative sacroiliac joint maneuvers and sacroiliac joint block are unreliable for diagnosing sacroiliac joint pain. Joint Bone Spine 2006;73(01):17-23

51 Young S, Aprill C, Laslett M. Correlation of clinical examination characteristics with three sources of chronic low back pain. Spine J 2003;3(06):460-465 